\title{
Could Helicobacter pylori infection increase the risk of coronary heart disease by modifying serum lipid concentrations?
}

Seppo Niemelä, Tuomo Karttunen, Taina Korhonen, Esa Läärä, Riitta Karttunen, Markku Ikäheimo, Y Antero Kesäniemi

\begin{abstract}
Objective-To investigate the relation between Helicobacter pylori infection and coronary heart disease (CHD).

Design-A case-control study.

Setting-Northern Finland (about 650000 inhabitants).

Patients-116 patients with angiographically documented CHD and 116 controls matched for age and gender randomly recruited from the register of the Finnish Social Insurance Institute.

Main outcome measures-The odds ratio (OR) estimates for the association of $H$ pylori infection with CHD.

Results $-64 \%$ of the CHD patients and $53 \%$ of the controls were seropositive for $H$ pylori; the OR adjusted for age and gender was $1.5(95 \%$ confidence interval (CI) 0.9 to 2.5). An additional adjustment for the common risk factors of CHD, including lipid concentrations, in a logistic regression analysis produced an OR estimate of $1 \cdot 1(95 \%$ CI 0.6 to $2 \cdot 1)$. Among the controls, those who were $H$ pylori positive had significantly $(P=0 \cdot 03)$ higher concentrations of serum triglycerides than those who were $H$ pylori negative: the trend among the cases was similar, but non-significant. The concentrations of HDL cholesterol tended to be lower in those who were $H$ pylori positive than in those who were $H$ pylori negative, among both the cases and the controls.
\end{abstract}

Conclusions-The impact of $H$ pylori infection as an independent risk factor for CHD seems to be minor. On the other hand the results are consistent with the hypothesis that $H$ pylori infection might modify the serum lipid concentrations in a way that could increase the risk of CHD.

(Heart 1996;75:573-575)

Keywords: coronary heart disease, Helicobacter pylori, serum lipids

Helicobacter pylori infection and coronary heart disease (CHD) are common conditions in late middle and old age. $H$ pylori usually causes a lifelong infection of the gastric mucosa. ${ }^{1}$ In addition, $H$ pylor $i$ has been shown to cause systemic responses related to $\mathrm{CHD} .{ }^{2-5} \mathrm{H}$ pylori is a major cause of gastric ulcer disease, which coincides with CHD more frequently than expected. ${ }^{6}$ Recently, $H$ pylori infection turned out to be associated with CHD in cross sec- tional studies. ${ }^{47}$ The prevalence of $H$ pylori infection is dependent on the population studied, ${ }^{1}$ and the results of the studies may be confounded by other risk factors for $\mathrm{CHD}$ and $H$ pylori infection. ${ }^{18}$ We investigated the association of $H$ pylori infection with CHD in a casecontrol study by comparing a group of patients with angiographically documented CHD and a group of controls with a similar age and gender distribution.

\section{Patients and methods}

The case series originally consisted of 138 consecutive patients referred to the Department of Internal Medicine, Oulu University Hospital, Finland, for elective coronary angiography because of suspected CHD. The catchment area comprised Northern Finland, which has about 650000 inhabitants. We excluded one patient without a serum sample for serological determination of $H$ pylori and 21 on lipid-lowering medication. We studied 116 patients (96 men, 20 women, aged 35-65 years, median 54 years) with angiographically documented coronary artery disease and 116 people (aged 40-59 years, median 52 years) randomly recruited from the register of the Finnish Social Insurance Institute covering the population of Oulu, the biggest city in the area, and matched for age and gender with the cases. The controls had not needed medical treatment for hypertension.

The frozen sera of the patients and the controls were simultaneously investigated for IgG antibodies to $H$ pylori by an enzyme linked immunosorbent assay (Pyloriset, Orion Diagnostica, Espoo, Finland) by one of the authors (RK) who was blind to the disease status. Titres $\geqslant 500$ were regarded as positive (a specificity and sensitivity of $95 \%{ }^{9}$ ). Fasting serum total cholesterol, HDL cholesterol, and total triglycerides were also measured. The patients and the controls were interviewed about their history of diabetes and smoking habits. The controls were also asked about their possible history of CHD. Selective coronary angiography was performed on the patients using the Judkins method and analysed according to the clinical routine of the hospital. A narrowing of the luminal dimensions of $50 \%$ or more was defined as a significant lesion. The patients were defined as having one, two, or three vessel disease, according to the number of arteries affected.

Concentrations of serum lipids (log transformed values) in the $H$ pylori positive and 
Table 1 Distribution of some determinants of coronary heart disease in patients with CHD and their controls who were seropositive or seronegative for Helicobacter pylor

\begin{tabular}{|c|c|c|c|c|}
\hline & \multicolumn{2}{|l|}{$C H D$ patients } & \multicolumn{2}{|l|}{ Controls } \\
\hline & Positive $(n=74)$ & Negative $(n=42)$ & Positive $(n=62)$ & Negative $(n=54)$ \\
\hline $\begin{array}{l}\text { Cholesterol (mmol/l) (median and quartiles) } \\
\text { HDL cholesterol (mmol/l) (median and quartiles) } \\
\text { Triglycerides (mmol/l) (median and quartiles) } \\
\text { Number with diabetes } \\
\text { Number ever smokers }\end{array}$ & $\begin{array}{l}6 \cdot 26(5 \cdot 6,7 \cdot 1) \\
0 \cdot 97(0 \cdot 9,1 \cdot 2) \\
1 \cdot 87(1 \cdot 4,2 \cdot 5) \\
4 \\
58\end{array}$ & $\begin{array}{l}6 \cdot 46(5 \cdot 5,7 \cdot 2) \\
1 \cdot 06(0 \cdot 8,1 \cdot 3) \\
1 \cdot 75(1 \cdot 1,2 \cdot 3) \\
4 \\
31\end{array}$ & $\begin{array}{l}5 \cdot 71(4 \cdot 9,6 \cdot 7) \\
1 \cdot 18(1 \cdot 0,1 \cdot 4) \\
1 \cdot 50(1 \cdot 1,1 \cdot 9) \\
2 \\
41\end{array}$ & $\begin{array}{l}5 \cdot 64(5 \cdot 2,6 \cdot 2) \\
1 \cdot 32(1 \cdot 1,1 \cdot 5) \\
1 \cdot 17(0 \cdot 9,1 \cdot 5)^{\star} \\
1 \\
28\end{array}$ \\
\hline
\end{tabular}

$\star \mathrm{P}=0.03$ for comparison between seropositive and seronegative individuals for log triglyceride concentrations adjusted for age, gender, and history of smoking in non-diabetic subjects.

negative individuals were compared in the control group, excluding those with a history of diabetes or some evidence of $\mathrm{CHD}$ and adjusting for age, gender, and history of smoking (current $v$ ex- and never-smokers) by the analysis of covariance. A similar analysis was performed on the CHD patients. The odds ratio (OR) for the relative risk of CHD associated with the $H$ pylori infection was estimated by the logistic regression model, adjusting by forward steps for age and gender; the history of smoking and diabetes; and total cholesterol, triglycerides and HDL cholesterol. Each lipid variable was divided into tertiles according to the distribution of values in the whole study group. We also analysed the data by comparing the patients with two or three vessel disease with the control group and by excluding the subjects with some evidence of CHD from the control group. The statistical analyses were performed using the SAS program (SAS Institute, Cary, NC, USA).

\section{Results}

Compared with the control group, the CHD patients had higher serum concentrations of total cholesterol and total triglycerides and lower serum concentrations of HDL cholesterol and were more often diabetic and eversmokers (table 1). In the control group, after adjustment for the effect of age, gender, and smoking by the analysis of covariance, those who were $H$ pylori positive had higher serum concentrations of triglycerides $(P=0.03)$ and lower HDL cholesterol $(P=0 \cdot 19)$ than those who were not. Findings in the CHD patients were similar $(P=0.18$ and $P=0 \cdot 18$, respectively).

Seventy four (64\%) cases and $62(53 \%)$ controls were $H$ pylori positive. The proportion of $H$ pylori-positive individuals was higher among the cases with two or three vessel disease $(51 / 74,69 \%)$ than in the other CHD patients $(23 / 42,55 \%)$. The results of logistic

Table 2 Estimated relative odds ratio (OR) with 95\% confidence interval (CI) of $H$ pylori positivity among all the patients with CHD and those with two or three vessel disease compared with controls and adjusted in a forward manner for certain determinants of CHD in a logistic regression models

\begin{tabular}{|c|c|c|c|c|}
\hline & \multicolumn{2}{|c|}{ All $C H D$} & \multicolumn{2}{|c|}{2 and 3 vessel disease } \\
\hline & $O R$ & $(C I)$ & $O R$ & $(C I)$ \\
\hline $\begin{array}{l}\text { (1) Age and gender } \\
\text { (2) } 1+\text { diabetes and smoking } \\
\text { (3) } 2+\text { total cholesterol } \\
\text { (4) } 3+\text { triglycerides } \\
\text { (5) } 4+\text { HDL cholesterol }\end{array}$ & $\begin{array}{l}1.48 \\
1.37 \\
1.40 \\
1.31 \\
1 \cdot 10\end{array}$ & $\begin{array}{l}(0 \cdot 9,2 \cdot 5) \\
(0 \cdot 8,2 \cdot 4) \\
(0 \cdot 8,2 \cdot 5) \\
(0 \cdot 7,2 \cdot 4) \\
(0 \cdot 6,2 \cdot 1)\end{array}$ & $\begin{array}{l}1 \cdot 86 \\
1.71 \\
1.62 \\
1.55 \\
1 \cdot 40\end{array}$ & $\begin{array}{l}(1 \cdot 0,3 \cdot 5) \\
(0 \cdot 9,3 \cdot 3) \\
(0 \cdot 8,3 \cdot 2) \\
(0 \cdot 8,3 \cdot 2) \\
(0 \cdot 6,3 \cdot 0)\end{array}$ \\
\hline
\end{tabular}

regression modelling (table 2) indicated that the CHD status was associated, though not significantly, with $H$ pylori positivity when adjusted for age and gender only $(\mathrm{OR}=1 \cdot 5)$. However, when further adjustments where made for diabetes, smoking, total cholesterol and triglycerides, the association became weaker $(\mathrm{OR}=1 \cdot 3$ ) and almost disappeared after adjustment for HDL cholesterol (OR = $1 \cdot 1)$. The relative risks were higher when the patients with two or three vessel disease were compared with the control group, but even then the association was fairly weak after adjustment for all of the above determinants and the confidence interval was wide. The results were similar when the analyses were performed by excluding the subjects with any evidence of CHD from the control group.

\section{Discussion}

Our CHD patients, especially those with two or three vessel diseases, were more often $H$ pylori seropositive than the non-diseased controls. Apart from the large random variation, the estimated odds ratios may be biased by the selection of the controls and the cross sectional study design. Our controls were representative of the source population of the cases, but the exclusion of the patients with obvious hypertensive disease reduced their risk of CHD. We found that $53 \%$ of our controls were seropositive for $H$ pylori, which accords with the previously reported seroprevalences of $39-60 \%$ among the Finnish blood donors aged 36-65 years. ${ }^{10}$ Some misclassification of infection status is likely. This will affect all the groups and hence tend to reduce the relative risk estimates towards 1 .

In the present study, the adjustment for the conventional risk factors, especially the serum HDL cholesterol concentration, reduced the estimated relative risk of CHD associated with $H$ pylori positivity. In a recent study of 47 subjects with electrocardiographic evidence of myocardial ischaemia or infarction and 341 controls, $H$ pylori infection was significantly associated with $\mathrm{CHD}$, and the relation could not be explained by the risk factors of CHD including history of hyperlipidaemia. ${ }^{4}$ However, the data also suggest a negative, although not statistically significant, effect of $H$ pylori positivity on plasma apolipoprotein A concentration (reflecting HDL cholesterol concentration) and a positive effect on triglyceride concentration. ${ }^{4}$ Another recent study reported a weak negative association between $H$ pylori infection and HDL-cholesterol. ${ }^{11}$ In addition, 
acute infections have been shown to reduce the serum HDL cholesterol concentration and increase the serum triglyceride concentration. ${ }^{12}$ In our study the similar trend observed both in the CHD patients and in the controls suggests that $H$ pylori may modify serum lipid concentrations. If this is true and the effect of $H$ pylori infection on the risk of CHD is mediated by HDL cholesterol and triglyceride concentrations, then these lipid variables should not be adjusted when the effect of $H$ pylori on the disease is assessed. If, however, $H$ pylori infection does not affect serum HDL cholesterol or triglyceride concentrations, these variables would be ordinary confounders and should be adjusted for. We analysed the data both without and with these adjustments and present the appropriate results for the alternative theories about the role of HDL cholesterol and triglycerides.

$H$ pylori infection and CHD may have shared risk factors, because low socioeconomic status in childhood may predispose to both conditions. ${ }^{18} \mathrm{We}$ did not have this information for those in our study. In the British study, adjustment for the features of current and childhood socioeconomic conditions only slightly reduced the effect of $H$ pylori seropositivity on the risk of $\mathrm{CHD}^{7}$ and a recent study concluded that the social class was associated with CHD independently of $H$ pylori infection. ${ }^{11}$

The present results indicate that after adjustment for some known key determinants of $\mathrm{CHD}, H$ pylori infection has little impact as an independent risk factor for CHD. However, the confidence intervals were wide leaving room for the possibility that $H$ pylori could have a moderate effect on the risk of CHD. Also the data are consistent with the hypothesis that $H$ pylori may modify serum lipid concentrations in a way that may increase the risk of CHD. A longitudinal study with treatment intervention and with more expected cases would be needed to examine the causal relations between these associations.

Orion Diagnostica, Espoo, Finland, provided the Pyloriset kits free of charge.

1 Megraud F. Epidemiology of Helicobacter pylori infection. Gastroenterol Clin North Am 1993;22:73-88.

2 Karttunen T, Niemelä S. Increased blood leucocytes in patients with Campylobacter pylori (letter). Ann Intern Med 1990;112:232.

3 Miragliotta G, Del Prete R, Mosca A. Helicobacter pylor infection and coronary heart disease. Lancet 1994;344: 751

4 Patel P, Mendall MA, Carrington D, Strachan DP, Leatham E, Molineaux N, et al. Association of Helicobacter pylori and Chlamydiae pneumoniae infections with corory pry BrMed f 1995:311;711-4.

5 Yarnell JWG, Baker IA, Sweetnam PM, Bainton D, O'Brien JR, Whitehead PJ, et al. Fibrinogen, viscosity, and white blood cell count are major risk factors for and white blood cell count are major risk factors
ischaemic heart disease. Circulation 1991;83:836-44.

6 Sonnenberg A. Concordant occurrence of gastric and onnenberg A. Concordant occurrence of gastric hypertensive diseases. Gastroenterology 1988;95:42-8.

7 Mendall MA, Coggin PM, Molineaux N, Levy J, Toosy T, Strachan $\mathrm{D}$, et al. Relation of Helicobacter pylori infection and coronary heart disease. Br Heart $f$ 1994;71:

8 Nilsson PM, Möller L, Östergren PO. Social class and cardiovascular disease-an update. Scand $\mathcal{F}$ Soc Med 1995 23:3-8

9 Feldman RA, Deeks JJ, Evans SJW. Multi-laboratory comparison of eight commercially available Helicobacter pylor serology kits. Eur $\mathcal{F}$ Clin Microbiol Infect Dis 1995;14 428-33.

10 Kosunen TU, Höök J, Rautelin HI, Myllylä G. Age-dependent increase of Campylobacter pylori antibodies in blood donors. Scand $\mathcal{f}$ Gastroenterol 1989;24:110-4

11 Murray LJ, Bamford KB, O Reilly DPJ, McCrum EE, Evans AE. Helicobacter pylori infection: relation with cardiovascular risk factors, ischaemic heart disease, and social class. Br Heart $\mathcal{F}$ 1995;74:497-501.

12 Sammalkorpi K, Valtonen V, Kerttula Y, Nikkilä E, Taskinen M-R. Changes in serum lipoprotein pattern induced by acute infections. Metabolism 1988;37:859-65. 